\title{
Beyond Einstein: Exploring the Extreme Universe
}

\author{
Louis M. Barbier* \\ NASA/Goddard Space Flight Center, Greenbelt, MD, 20771
}

\begin{abstract}
This paper will give an overview of the NASA Universe Division Beyond Einstein program. The Beyond Einstein program consists of a series of exploratory missions to investigate some of the most important and pressing problems in modern-day astrophysics including searches for Dark Energy and studies of the earliest times in the universe, during the inflationary period after the Big Bang. A variety of new technologies are being developed both in the science instrumentation these missions will carry and in the spacecraft that will carry those instruments.
\end{abstract}

\section{Introduction}

$\mathrm{I}^{\mathrm{t}}$ has been nearly 100 years since Einstein's theory of relativity was published. The world has changed dramatically since then, in a large part due to the bold, revolutionary ideas put forward by Einstein. As we begin another century of exploration - including the vision of putting men on Mars - we anticipate equally bold revolutions in our understanding of the universe. This talk will attempt to summarize the NASA Universe Division exploration program, Beyond Einstein.

Beyond Einstein will employ the newest technology in a variety of areas, to bring us to the most extreme places in the universe - the very early universe after the Big Bang, edges of black holes, the warped accretion disks surrounding them, and the almost unimaginable collisions between them. The two great observatories that form the nucleus of the Beyond Einstein program are LISA and Constellation-X. LISA (the Laser Interferometer Space Antenna) will produce the first images ever of the universe in gravitational waves. Gravitational waves, predicted by relativity theory but never directly observed, are emitted by many types of objects - especially compact binaries, in neutron star - neutron star collisions, black hole collisions, and even from the inflation of the universe itself. LISA will be the first space-based gravitational wave detector, deploying three spacecraft in an interferometer configuration, over a separation of five million kilometers. LISA will require new techniques and technologies in laser science and formation flying to achieve its goals.

Constellation- $X$ will provide new precision measurements in the $\mathrm{x}$-ray regime - with extended energy range, improved resolution, and higher sensitivity than current missions. Constellation- $X$ will measure the velocities and conditions of material moving in the extreme gravitational potentials outside black holes - in the accretion disks that form outside them. With unprecedented sensitivity, it will trace the evolution of supermassive black holes over cosmic time. Constellation- $X$ will require advanced $\mathrm{x}$-ray $\mathrm{CCD}$ arrays, high throughput $\mathrm{x}$-ray optics, and superconducting microcalorimeter arrays.

These missions will begin the Beyond Einstein program, making new discoveries and focusing on areas where Einstein's theories left off. They will set the stage for a series of focused scientific probes: the Black Hole Finder probe - which will survey the universe and map out the black hole distribution in low energy gamma rays and hard $\mathrm{x}$-rays; the Inflation Probe will map the polarization of the Cosmic Microwave Background to search for the effects of gravitational waves and inflation; and the Dark Energy Probe which will map out the mysterious dark energy recently discovered to be pulling the matter in the universe apart!

I will briefly discuss these missions and the technologies that will be needed to carry them out and that will lead to the Beyond Einstein Vision Missions- the Big Bang Observer and the Black Hole Imager. Other talks will cover these in more detail.

\footnotetext{
" Astrophysicist, Astroparticle Physics Laboratory, Code 661.
} 


\section{The Great Observatories}

Two observatory class missions will lead the Beyond Einstein program, LISA and Constellation-X. Each will be featured in a separate talk in this session. LISA will be a Michelson interferometer comprised of three spacecraft separated by $\sim 5$ million kilometers. Each spacecraft will house a 30-cm telescope to direct the beam of a 1-micron cavity-stabilized laser toward the other spacecraft. The light received from each of the two other spacecraft is combined to look for "beats" between the local laser and the distant ones. These beats are a signal of the passage of gravitational waves through our solar system - causing the separation of the spacecraft to vary as space itself is distorted by the gravitational radiation. LISA will be sensitive to strains as small as $6 \times 10^{-21}$, or distances of $3 \times 20^{-}$

${ }^{12}$ meters. LISA will observe the entire sky at once and be able to detect a variety of sources by their distinct timevarying signatures. In looking for example at supermassive black hole mergers from distant galaxies, LISA will detail for us for the first time violently knotted spacetime regions that will severely test and constrain general relativity, and may show regions where it breaks down.

Each LISA spacecraft will house a "gravitational reference unit" consisting of a proof-mass and a sensitive position-measuring device. Micro-newton thrusters will be required to maintain the spacecraft in a drag-free orbit about the proof masses.

Constellation- $X$ will dramatically increase our sensitivity in the $\mathrm{X}$-ray range - more than 100 times the sensitivity of Chandra. This unprecedented sensitivity will enable us to constrain the nature of dark matter and dark energy by observing their effects on the formation of clusters of galaxies. It will enable us to determine the properties of matter at nuclear densities by measuring spectral features from the surfaces of neutron stars and to track the motion of hot plasmas at the edges of black holes.

Constellation-X will consist of four 1.6-meter $\mathrm{X}$-ray telescopes with grazing incidence optics. Each telescope will have a high-resolution $x$-ray spectroscopy system for imaging between $0.2-10 \mathrm{keV}$, and a low-resolution imager for hard $x$-rays, $10-60 \mathrm{keV}$. The spectroscopy systems will have 15 arcsec resolution in a $2.5 \mathrm{arcmin}$ field, and the hard $x$-ray telescope will have 1 arcmin resolution in an 8 arcmin field.

\section{The Beyond Einstein Probes}

Three probes are planned for Beyond Einstein, each focusing on a specific science question. The first probe will be the Dark Energy Probe. Very recently we have discovered that the universe is filled with a "dark energy" - an unseen field that comprises $70 \%$ of the universe and is causing the universe to expand at an increasingly faster rate. No one yet knows what this mysterious dark energy is. The Dark Energy Probe will attempt to answer this most important question by making optical and infra-red observations of the geometry of the universe in the $z \sim 0.7-2$ range. Several options are being explored. One approach is to obtain a large sample of Type Ia supernovae out to high redshifts.

The Inflation Probe will detect the signature of gravitational waves from the inflation of the universe, by measuring the polarization signal imprinted onto the cosmic microwave background. The polarization is expected to be an order of magnitude weaker than the polarization induces by quantum fluctuations. Large arrays of polarization-sensitive detectors, operating between $50-500 \mathrm{GHz}$ are required, operating at $100 \mathrm{mK}$.

The third probe is the Black Hole Finder Probe which will conduct a wide-field survey of black holes. Hard xray / soft gamma-rays should be able to penetrate the gas and dust surrounding black holes, and provide 5 arcmin position resolution. To achieve such a large survey, a large (several square meters) focal plane of relatively large (mm size) pixels would be used. A search in this regime would yield a sky map of black holes which would be further studied by other Beyond Einstein missions - Constellation-X, Black Hole Imager, LISA for example.

\section{The Vision Missions}

All of the previous missions, as described lead up to the two Vision Missions - Big Bang Observer and Black Hole Imager. These Vision Missions will produce dramatic scientific results - producing movies of material flowing in the accretion disks and highly contorted spacetime around black holes and observing the quantum fluctuations of the gravitational radiation from the period of inflation after the Big Bang. Both missions are under study.

Black Hole Imager will consist of a constellation of 27 spacecraft, spaced $\sim 1 \mathrm{~km}$ apart, with highly evolved formation flying - keeping them positioned to within roughly 5 microns. The expected angular resolution of the instrument will be $\sim 0.1$ micro-arcsec. Precise metrology will also be required for all the spacecraft and optical components, whose positions should be known to nm precision over several hours.

Big Bang Observer will follow LISA in detection of gravitational waves. Where LISA will use 3 spacecraft separated by $\sim 5$ million $\mathrm{km}, \mathrm{BBO}$ will use 9 spacecraft, $50,000 \mathrm{~km}$ apart to sample a different time domain 
compared to LISA, seconds instead of hours. BBO will need a strain sensitivity 10,000 times better than LISA, GRUs with 100 times lower noise, and laser power of $\sim 300$ Watts.

\section{Conclusion}

While the Beyond Einstein program is an ambitious one - the rewards it offers are stupendous. It offers a tremendous advance in our knowledge of the current universe and its contents, the very early universe and the inflation period that followed the Big Bang, and the future of our Universe. We can look forward to catalogs of black holes, direct measurements of the material surrounding them, movies of their motion, correlations of gravitational radiation and x-ray from a variety of sources, and a detailed polarization map of the CMB. Another revolution is coming, and NASA's Beyond Einstein program will help to usher it in.

\section{Acknowledgments}

The author thanks the AIAA for the invitation to attend this conference and present this talk. 